\title{
CT and MRI findings of branchial cleft cysts
}

\author{
Erkan Gökçe, Murat Beyhan* \\ Tokat Gaziosmanpasa University Faculty of Medicine, Department of Radiology, Tokat, Turkey
}

\begin{abstract}
Our aim in this study was to evaluate the CT and MRI findings of branchial cleft cysts (BCCs).

The demographic characteristics of patients who were found to have BCC in their neck radiological examinations were evaluated retrospectively. The dimensions and localizations of the BCCs, and the presence of septation and ruptures in the cysts were examined. Lesion density on $\mathrm{CT}$ and $\mathrm{T} 1$ - and $\mathrm{T} 2$-weighted signal properties compared to the sternocleidomastoid muscle on MRI of the lesions, as well as their contrast-enhancement patterns, were evaluated. First BCCs were subclassified based on Work classification system while Bailey classification was used to subclassify second BCCs.

BCC was observed in 16 cases (10 female and 6 male). The mean age of the cases with BCC was $28.4 \pm 15.0$ years. Fifteen of the BCCs were second BCC while one was first BCC. The only first BCC was Type 1 pattern based on Work classification. According to Bailey classification, 13 of the second BCCs had Type 2, one had Type 1 and one had Type 3 pattern. BCC diameters varied from 12 to $60 \mathrm{~mm}$. Mean density of the BCCs was 33.5 $\pm 12.6 \mathrm{HU}$. On MRI, BCCs were mostly hyperintense on T1- and T2-weighted images. Peripheral enhancement was detected in 12 BCCs. Septation was observed in three BCCs while one of them was ruptured. BCCs are more frequently observed in female and on the right side of the neck. They mostly have second BCC pattern. Radiologically, BCCs are cysts with different densities which can have peripheral enhancement, and they rarely have septations and ruptures.
\end{abstract}

Key Words: Branchial anomaly, Branchial cleft, Branchial cleft cyst, Computed tomography, Magnetic resonance imaging

\section{Introduction}

Branchial cleft anomalies are the most common congenital neck pathologies in the lateral neck and are caused by the absence of loss of branchial clefts that should be regressed during fetal development. Branchial cleft cysts (BCCs) develop from the first or second, only rarely from the third, branchial clefts in the fourth-seventh weeks of embryonic life. Second BCCs are most commonly observed (95\%) branchial anomaly. BCCs are soft, painless and mobile lesions, and might have variable sizes (1). They usually manifest themselves from the first to the fourth decades of the life (2). The late appearance of the BCCs depends on their growth as a result of upper respiratory tract infection. Dyspnea, dysphagia and dysphonia may occur depending on the size of the BCC. If a BCC is ruptured, discharge may develop during infection (3). Clinical and radiological findings are important in preoperative diagnosis to demonstrate the characteristics of lesion (4). Radiological examinations are important in determining the localization of the cyst, the path and the neighborhoods followed by the tractus (5). In the present study, radiological imaging findings of BCCs detected on computed tomography (CT) or magnetic resonance imaging (MRI) examinations were evaluated.

\section{Materials and Methods}

BCC cases were identified among the patients who were evaluated in our Radiology Department between 2007 and 2019 with the complaints of mass, pain, swelling, etc. in the neck and had BCC diagnosis based on neck CT, MRI and histopathological examinations. Eight cystic lesions which were diagnosed as BCC radiologically but not histopathologically because they were not operated were excluded from the study. Six of all operated patients were reported histopathologically as infected BCC.

Since the study was retrospective, the examinations performed on patients could not be standardized. The images of nine patients who had CT were taken using 4- or 8-detector CT devices and with a slice thickness of $3.75-5.00 \mathrm{~mm}$. The

\footnotetext{
*Corresponding Author: Murat Beyhan, Department of Radiology, Tokat Gaziosmanpasa University Faculty of Medicine, Kaleardı Mah., Muhittin Fisunoglu Cad., Tokat 60100, Turkey

E-mail: m_termeli@hotmail.com, Phone: 0 (535) 83927 16, Fax: 0 (356) 2133179

ORCID ID: Erkan Gökçe: 0000-0003-3947-2972, Murat Beyhan: 0000-0002-8630-4632

Received: 16.06.2020, Accepted: 09.12.2020
} 

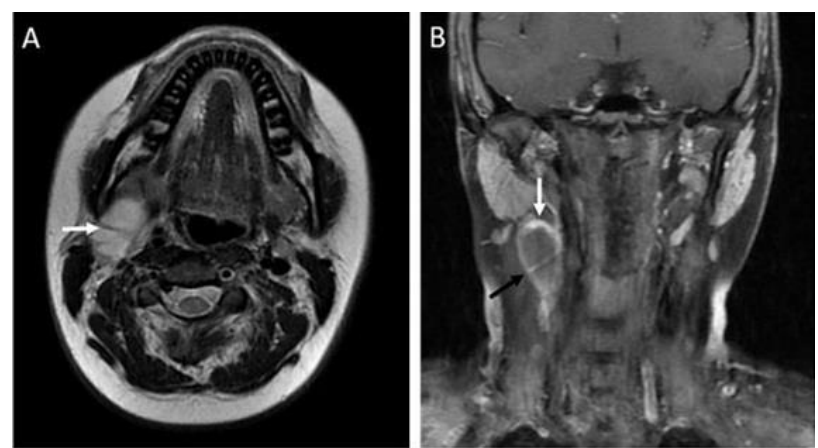

Fig. 1. Second branchial cleft cyst observed on magnetic resonance examination of an 18-year-old female patient; A) on axial T2-weighted image, cyst with homogeneously hyperintense signal and thin walls (white arrow) indented into the gland between the right sternocleidomastoid muscle and submandibular gland is shown; B) slight thickening in the superior wall of the cyst (white arrow) and contrast-enhancement in the thin septation extending into the cyst (black arrow) are shown on coronal T1-weighted image passing through the cyst posterior

MRI images of nine patients who were subjected to MRI examination were taken with a $1.5 \mathrm{~T}$ MRI device using head and neck array coils. Two of the patients had both CT and MRI, and the time between the two examinations was no longer than nine days. Ultrasonography (US) image was also available for a patient who had MRI examination. MR sequences included axial T1W sequence (TR: $600 \mathrm{~ms}$, TE: $10.1 \mathrm{~ms}$, NEX: 1, slice thickness: 6 $\mathrm{mm}$, gap distance: $1.5 \mathrm{~mm}$, FOV: $22 \mathrm{~cm}$ ), in phase axial T2W fat saturation (FATSAT) sequence (TR: $4340 \mathrm{~ms}$, TE: $87.7 \mathrm{~ms}$, NEX: 2, slice thickness: 6 $\mathrm{mm}$, gap distance: $1.5 \mathrm{~mm}$, FOV: $22 \mathrm{~cm}$ ), sagittal short tau inversion recovery (STIR) sequence (TR: $5120 \mathrm{~ms}$, TE: $35.7 \mathrm{~ms}$, NEX: 0.5, slice thickness: $5 \mathrm{~mm}$, gap distance: $1 \mathrm{~mm}$, FOV: 22 $\mathrm{cm}$ ) or sagittal T2W sequence (TR: $3680 \mathrm{~ms}$, TE: $105 \mathrm{~ms}$, NEX: 0.5, slice thickness: $4 \mathrm{~mm}$, gap distance: $1 \mathrm{~mm}$, FOV: $25 \mathrm{~cm}$ ) and coronal T2W sequence (TR: $3880 \mathrm{~ms}$, TE: $110 \mathrm{~ms}$, NEX: 1, slice thickness: $4 \mathrm{~mm}$, gap distance: $1 \mathrm{~mm}$, FOV: 24 $\mathrm{cm})$. Contrast-enhanced MRI was performed with axial and coronal T1W FATSAT sequence (TR: $560 \mathrm{~ms}$, TE: $9.06 \mathrm{~ms}$, NEX: 2, slice thickness: 4 $\mathrm{mm}$, gap distance: $1 \mathrm{~mm}$, FOV: $24 \mathrm{~cm}$ ) coronal (TR: $560 \mathrm{~ms}$, TE: $9.06 \mathrm{~ms}$, NEX: 2, slice thickness: $4 \mathrm{~mm}$, gap distance: $1 \mathrm{~mm}$, FOV: 24 $\mathrm{cm})$.

The demographic characteristics of the patients found to have BCC were studied. Images of the patients were evaluated on a consensus basis by two radiologists (E.G. with more than ten years of experience and M.B. with more than five years of experience in neuroradiology) using the hospital's
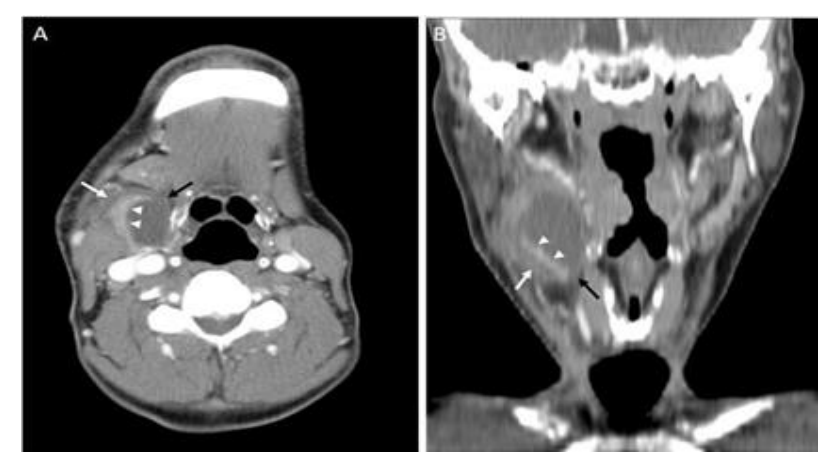

Fig. 2. A 53-year-old male patient who underwent a contrast-enhanced computed tomography examination; A) axial; B) coronal plane of contrast-enhanced, infected second branchial cleft cyst with thickened inferolateral wall (arrowheads), which developed liquid leak (white arrows) around it as a result of rupture at the antero-inferomedial part (black arrow)

picture archiving and communication system (Sectra IDS7 PACS, Sweden). On CT or MRI examinations, the anteroposterior (AP), mediolateral (ML) and craniocaudal (CC) dimensions of BCCs were measured, and presence of septation and rupture in the cyst was determined. Contrast material was used on CT and MRI examinations of all cases. On CT examination, the density of the lesion as Hounsfield unit (HU) was measured, and contrastenhancement pattern was evaluated. On conventional MRI sequences, the signal characteristics and contrast-enhancement pattern of the lesions in T1-weighted and T2-weighted sequences were evaluated compared to sternocleidomastoid muscle. HU density of the lesion was measured from the widest part of the cystic area with a single region of interest measurement. Cyst type (Type 1 and 2 patterns) were examined based on Work classification in first BCCs. In the second BCCs, the cyst types (Type 1, 2, 3 and 4 patterns) were evaluated based on the Bailey classification. The study was retrospective and approved by the local ethics committee (20-KAEK-104).

Statistical Analyses: Our study had a descriptive design, and demographic characteristics of the cases were examined. The quantitative variables were presented as mean and standard deviation while qualitative variables were given as percent. Statistical analyses were performed using SPSS software (IBM SPSS Statistics 22, SPSS Inc., an IBM Co., Armonk, NY, United States).

\section{Results}

BCCs were detected in a total of 16 patients, 10 female $(62.5 \%)$ and 6 male $(37.5 \%)$. The mean age 

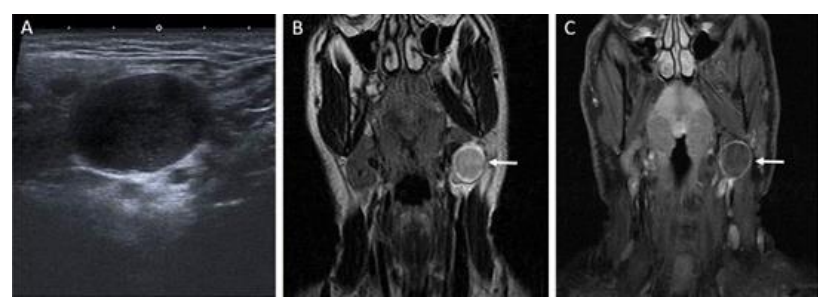

Fig. 3. Forty-two-year-old male patient found to have infected second BCC; A) On ultrasonography, thin-walled, smooth-contoured cystic lesion with fine internal echogenicities due to dense content is shown; B) on the coronal T2-weighted magnetic resonance image, the periphery of the mass showed a more hyperintense signal (arrow) compared to the muscle; C) on contrast-enhanced coronal T1-weighted magnetic resonance image, thin rim-like contrast-enhancement (arrow) of the cystic mass is shown

of the patients with the BCC was $28.4 \pm 15.0$ (range: 9-53). BCCs were localized on the right in 12 patients $(75 \%)$ and on the left in 4 patients (25\%). Minimum and maximum BCC diameters were 12 and $60 \mathrm{~mm}$, respectively. Mean BCC dimensions were $31.8 \pm 8.8 \mathrm{~mm}$ in AP, $27.1 \pm 6.9$ $\mathrm{mm}$ in $\mathrm{ML}$ and $39.3 \pm 11.6 \mathrm{~mm}$ in CC directions. The average density of BCCs was $33.5 \pm 12.6 \mathrm{HU}$ (range: 17-60 HU) in 9 patients who had CT examinations. The mean density of the 3 infected BCCs was $32.3 \pm 8.3 \mathrm{HU}$ (range: 27-42 HU) while the mean density of the 6 non-infected BCCs was $34.1 \pm 15.0 \mathrm{HU}$ (range: 17-60 HU). In 9 patients who underwent MRI, 7 were hyperintense and 2 were isointense on T1-weighted sequences while all of them were hyperintense on $\mathrm{T} 2$-weighted sequences. On contrast-enhanced MRI and CT, 12 BCCs had peripheral rim-like enhancement, and 8 of these lesions also had thick-walled contrastenhancement, which was focal in places. Contrastenhancement was not observed in 4 of the BCCs. Septation was detected in three BCCs (Figure 1) while one had cyst rupture (Figure 2). In a case which also underwent US examination, a cyst lesion with dense content, thin-wall and smoothcontour was observed (Figure 3). No changes were observed in the nature of BCCs (size increase, septation or rupture) possibly because the number of patients who had both MRI and CT examinations was limited (two patients) and the time between the examinations was quite short.

Fifteen of the cases were second BCC while one was first BCC. Third and fourth BCCs were not encountered in the present study. The only first BCC (Figure 4) was classified as Type 1 pattern based on Work classification. Thirteen of the 15 second BCCs (86.7\%) had Type 2, while one

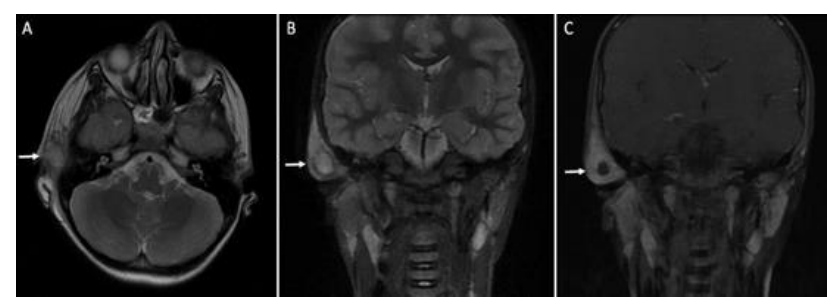

Fig. 4. Magnetic resonance examination of a 9-year-old male patient with the first branchial cleft cyst infected in the right preauricular region; A) axial; B) coronal T2weighted images. Mildly heterogeneously hyperintense, thick-walled cyst (arrows) is shown; C) on contrastenhanced coronal image, contrast-enhancement secondary to the infection-inflammation in the cyst wall and in adjacent soft tissues are shown (arrow)

(6.7\%) had Type 1 and one (6.7\%) had Type 3 pattern based on Bailey classification (Figure 5).

\section{Discussion}

Although BCCs are generally congenital abnormalities that occur in childhood, they can also develop in adults as a mass or infection in the neck. In a study examining 62 patients with BCC, $64.5 \%$ of patients were female, $35.5 \%$ were male, and the mean age was $26.5 \pm 12.9$ years (6). Similar to the literature, $62.5 \%$ of BCC patients were female and $37.5 \%$ male in the present study, and the mean age was $28.4 \pm 15.0$ years. In another study examining 30 patients, $55 \%$ of BCCs were reported to be localized on the right side (7). In our study, $75 \%$ of the BCCs were on the right, which was somewhat higher than what was reported in the literature. In a study analyzing BCCs, cyst sizes were found between 20 and $95 \mathrm{~mm}$ (8). In the present study, BCC sizes varied from 12 to $60 \mathrm{~mm}$, and these values were somewhat low compared to the literature.

First BCCs are usually found as expanded masses close to the lower pole of the parotid gland, and are more common in middle-aged patients. They may be in an intraparotid or extraparotid locations. First BCCs occur as oval or round cystic masses, which can have variable wall thickness and contrastenhancement (9). The branchial cleft anomalies were categorized by Work into two patterns in 1972: Type 1 and Type 2. Those on the Type 1 pattern are entirely of ectodermal origin and are found in cystic masses adjacent to external auditory canal. They are duplications of membranous external auditory canal and contain squamous epithelium, but they do not contain skin adnexa or cartilages. Those in type 2 patterns can appear as cysts, sinuses or fistulas in the angle of the mandible region. They are ectodermal and mesodermal origin, so they contain squamous epithelium, skin adnexa (hair follicles, sweat and sebaceous glands) and cartilages. 


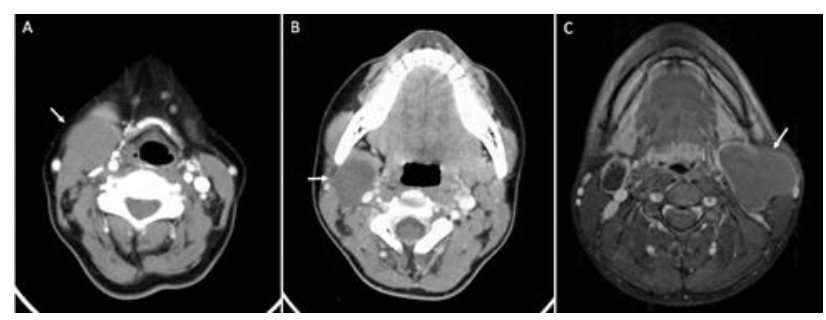

Fig. 5. A) Contrast-enhanced axial neck CT examination of a 53-year-old female patient shows branchial cleft cyst (Bailey Type 1 pattern) on the right side of the neck with a density of $60 \mathrm{HU}$; B) contrast-enhanced axial neck CT image shows a second branchial cleft cyst (Bailey Type 2 pattern) in a 15-year-old female patient with a density of 40 $\mathrm{HU}$ on the right side of the neck; C) contrast-enhanced axial neck MRI examination shows a second branchial cleft cyst (Bailey Type 3 pattern) on the left side of the neck in a 22-year-old male patient

Second BCCs account for 95\% of all BCCs (9). Second branchial cleft anomalies are most commonly found as cysts, followed by sinuses and fistulas (10). Fistulas and sinuses are usually observed before the age of 10 , while cysts are more common between the ages of 10 and 40 (9). Second BCCs are localized along the front edge of the sternocleidomastoid muscle in the upper part of the neck (1). Most of them are found in submandibular space, but the tonsils can appear anywhere along the second branchial arc, which extends from the skin above the supraclavicular fossa, to enter pharynx at the level of fossa. Second BCCs were classified by Bailey under four different patterns in 1929: Type 1 pattern is the most superficial one, and is located along the anterior surface of the sternocleidomastoid muscle, deep in the platysma, but not in contact with the carotid sheath. The type 2 pattern is the most common type found in front of the sternocleidomastoid muscle, on the posterior of the submandibular gland, next to and lateral to carotid sheath. The type 3 pattern extends to the pharyngeal wall lateral between the bifurcation of the internal and external carotid arteries in the medial. Type 4 pattern extends deep into the carotid sheath in the pharyngeal mucosal cavity, and opens into the pharynx (10).

Third BCCs are rare. However, they are most common congenital lesions of the posterior cervical region after thyroglossal cysts. Third BCCs are found characteristically in the depths of the sternocleidomastoid muscle. Fourth BCCs are also rare, and they constitute 1-4\% of all branchial cleft anomalies. Like third BCCs, they are commonly localized on the left side. Fourth BCCs are typically localized in the thyroid gland and mediastinum (11). In a study in which 42 of the 62 patients scored $(67.7 \%)$ had second BCCs and $20(32.3 \%)$ had first BCCs, 13 of the 20 patients with primary BCCs had
Type 1 and 7 patients had Type 2 patterns based on Work classification system (6). In another study dealing with 17 second BCCs, five patients were found to have Type 1 pattern and 12 patients had Type 2 pattern based on Bailey classification (12). In the present study, $93.7 \%$ of patients had second BCC, while third and fourth BCC were not observed. The most common pattern among second BCCs in the present study was Type 2 based on Bailey classification while Type 4 pattern was not observed in any of the patients. First BCC was found in only one case, which was of Type 1 pattern based on Work classification.

Radiological methods are helpful in the characterization of lesion in patients clinically suspected of have BCC through inspection and palpation. The radiological method which should be preferred first in the diagnosis of cysts is US because it is easy to reach and has no radiation exposure. US examination is often sufficient in the diagnosis of non-complicated BCCs (13). Well-bordered, hypoechoic or anechoic, thin-walled lesion with no intralesional septation on the US suggests BCC. No intralesional vascularization is observed on Doppler US $(2,14)$. However, cross-sectional imaging methods such as CT and MRI could be employed in the diagnosis of complicated BCCs. On CT, typically a well-bordered, thin-walled, uniformly hypodense cystic mass is detected when complication is not involved. BCCs have an attenuation value at water density $(<20 \mathrm{HU})$ on CT (9). BCCs might have variations between hypointense and mild hyperintense relative to the muscle on T1-weighted sequences of MRI, but they are usually hyperintense on T2A sequences $(2,14)$. On MRI, the hyperintensity of BCCs on T1-weighted sequences depends on the protein content of the cysts (15). In inflammatory events, the cyst wall thickens, and peripheral contrastenhancement increases (14). The mean density of the BCCs in the present study (33.5 HU) was higher than the values reported in the literature. The average density of non-infected BCCs was slightly higher than that of infected ones. In most cases who underwent MRI examination, BCCs were hyperintense on T1weighted sequences. Higher densities on CT and hyperintensity on T1-weighted sequences of MRI suggested that BCCs in the present study were dense and had protein contents. In addition, three-quarters of the patients found to have BCCs had peripheral rim-like contrast-enhancement. While septation was reported in a case with malignant transformation in BCC literature (12), three of our cases had septation in cysts. However, malignant transformation was not detected in any of our cases. MRI is superior to CT in that it does not involve radiation exposure and better 
shows the extension of cysts and accompanying abscess formations. However, the necessity of sedation on MRI in children younger than six years of age and the length of the procedure lead to higher preference for CT (13). Fine needle aspiration biopsy, fistulogram and pharyngeoesophagogram examinations are also helpful in the diagnosis of BCCs (5).

Systemic antibiotics play a minor role in the management of benign neck cysts if no infection occurs. Recently, minimally invasive treatment has become an alternative for the surgery. Symptomatic benign cysts can be managed with fine-needle aspiration drainage, although some of the cysts will recur. Percutaneous ethanol injection is other minimally invasive treatment procedure. The chemical effects of ethanol injection cause cellular dehydration, protein denaturation, hemorrhage infraction and result in fibrosis, and finally regression and shrinkage of the cysts (16). Curative treatment of BCCs is surgical excision (13). Complete excision of these lesions is required to prevent recurrence (17). Recurrent infections due to BCCs and fistula tracts secondary to infection could develop in the skin. Recurrent infections can make surgical treatment difficult, especially in BCC anomalies with intraparotid localizations. In the acute infections of third and fourth BCCs, airway obstruction and swallowing problems may occur due to pharyngeal edema. Management of BCCs begins with the infection control first, if any. After the control of the infection, cysts are often excised to prevent recurrent episodes of infection and the complications from these episodes (18).

Differential diagnosis of BCCs includes cystic hygroma, hemangioma, tuberculous lymphadenitis, dermoid cyst, sebaceous cyst, teratoma, thymic cyst, sarcoidosis, metastatic squamous cell carcinoma, HIV-related lymphadenitis, lymphoma and metastatic papillary carcinoma of thyroid (8, 19). Cystic hygroma can be observed as a pseudosolid heterogeneous hypoattenuated mass containing internal debris and septa $(2,11)$. Hemangiomas are often observed as lobular lesions with high signal intensities in T2-weighted images but in T1-weighted images they have intensities between fat and muscle (20). Tuberculosis lymphadenitis is observed on US as a thickly encapsulated, hypoechoic and multilocular mass, but on CT it appears as conglomerate nodal mass with low density in the center and irregular contrasting in the periphery. The same findings may be observed in lymphoma, metastatic carcinoma and HIV-related lymphadenitis $(21,22)$.
Dermoid and sebaceous cysts often appear as uniform, thin-walled, fat-containing cystic masses of water density. Teratomas are non-homogeneous masses that can contain calcification and adipose tissue (9). Thymic cysts are observed as unilocular or multilocular cystic masses with hypoattenuation adjacent to carotid space (2). About $40 \%$ of all lymph node metastases caused by papillary thyroid carcinoma tend to have complete cavitation and therefore can mimic a seemingly benign cervical cyst (9).

The first limitation of the present study was that radiological examinations could not be standardized in all cases due to retrospective nature of the study and relatively low number of patients (some cases had only neck CT and some neck MRI, while multiple examinations were performed in some cases). The second limitation was that since neck CT and MRI examinations were not performed for each case, imaging characteristics of the lesions based on imaging modality were not evaluated in every patient.

In conclusion, the BCCs are more common in female gender, and are more frequently localized on the right side of the neck. They frequently have second BCC patterns. On radiological imaging, BCCs could have different density or intensities depending on their contents. They often have thin peripheral rim-like contrast-enhancement, while septation and rupture can rarely be seen.

Conflict of interest: Authors declare no conflict of interest.

\section{References}

1. Tansuker HD, Coşkun BU, Sözen ME, Başak T, Dadaş B. Branchial cleft cyst: A rare diagnosis in a 84-year old patient. A case report. Turk Arch Otolaryngol 2011; 49: 22 24.

2. Koeller KK, Alamo L, Adair CF, Smirniotopoulos JG. Congenital cystic masses of the neck: radiologic-pathologic correlation. Radiographics 1999; 19: 121-146.

3. Enepekides DJ. Management of congenital anomalies of the neck. Facial Plast Surg Clin North Am 2001; 9: 131-145.

4. Papadogeorgakis N, Petsinis V, Parara E, et al. Branchial cleft cysts in adults. Diagnostic procedures and treatment in a series of 18 cases. Oral Maxillofac Surg 2009; 13: 79-85.

5. Daoud FS. Branchial cyst: an often forgotten diagnosis. Asian J Surg 2005; 28: 174-178. 
6. Baran H, Aydin S. Branchial Cleft Anomalies: Our Clinical Experience and Literature Review. Kocaeli Med J 2019; 8: 33-37.

7. Prasad SC, Azeez A, Thada ND, Rao P, Bacciu A, Prasad KC. Branchial anomalies: diagnosis and management. Int J Otolaryngol 2014; 2014: 237015.

8. Alimoğlu Y, Altın F. Second Branchial Cleft Anomalies: Analysis of Our Clinical Experience in 10 Years. KBB-Forum 2020; 19: 22-26.

9. Handa N, Taneja A, Atwal SS, Madhav RKV. Imaging of cystic neck masses in adults. Kulak Burun Bogaz Ihtis Derg 2017; 27: 151-157.

10. Adams A, Mankad K, Offiah C, Childs L. Branchial cleft anomalies: a pictorial review of embryological development and spectrum of imaging findings. Insights Imaging 2016; 7: 69-76.

11. Bagchi A, Hira P, Mittal K, Priyamvara A, Dey AK. Branchial cleft cysts: a pictorial review. Pol J Radiol 2018; 83: 204-209.

12. $\mathrm{Hu} \mathrm{S}, \mathrm{Hu} \mathrm{CH}$, Yang $\mathrm{L}$ et al. Atypical imaging observations of branchial cleft cysts. Oncol Lett. 2014; 7: 219-222.

13. Leung AK, Davies HD. Cervical lymphadenitis: Etiology, diagnosis, and management. Curr Infect Dis Rep 2009; 11: 183-189.

14. Valentino M, Quiligotti C, Carone L. Branchial cleft cyst. J Ultrasound 2013; 16: 17 20.

15. Som PM, Curtin HD. Head and Neck Imaging - Volume 2 (5th ed). St. Louis: Mosby Elsevier $2011 ; 2240$
16. Liao LJ, Wang CT, Huang TW, Cheng PW, Lo WC. Ultrasound-guided-fine-needle aspiration drainage and percutaneous ethanol injection for benign neck cysts. J Med Ultrasound 2020; Epub ahead of print, https://www.jmuonline.org/temp/JMedUltras ound000-5354484_145224

17. Sun Z, Fu K, Zhang Z, Zhao Y, Ma X. Multidetector computerized tomographic fistulography in the evaluation of congenital branchial cleft fistulae and sinuses. Oral Surg Oral Med Oral Pathol Oral Radiol 2012; 113: 688-694.

18. Enepekides DJ. Management of congenital anomalies of the neck. Facial Plast Surg Clin North Am 2001; 9: 131-145.

19. Hart C, Opperman D, Gulbahce E, Adams G. Branchial cleft cyst: A rare diagnosis in a 91 year-old patient. Otolaryngol Head Neck Surg 2006; 135: 955-957.

20. Vilanova JC, Barceló J, Smirniotopoulos JG, et al. Hemangioma from head to toe: MR imaging with pathologic correlation. Radiographics 2004; 24: 367-385.

21. Bayaz1t YA, Bayaz1t N, Namiduru M. Mycobacterial cervical lymphadenitis. ORL J Otorhinolaryngol Relat Spec 2004; 66: 275280.

22. Herts BR, Megibow AJ, Birnbaum BA, Kanzer GK, Noz ME. High-attenuation lymphadenopathy in AIDS patients: significance of findings at CT. Radiology 1992; 185: 777-781. 